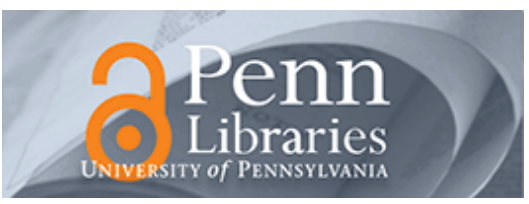

University of Pennsylvania

ScholarlyCommons

April 2004

\title{
Peculiar Radar Cross-Section Properties of Double-Negative and Single-Negative Metamaterials
}

\author{
Andrea Alù \\ University of Pennsylvania, andreaal@seas.upenn.edu \\ Nader Engheta \\ University of Pennsylvania, engheta@seas.upenn.edu
}

Follow this and additional works at: https://repository.upenn.edu/ese_papers

\section{Recommended Citation \\ Andrea Alù and Nader Engheta, "Peculiar Radar Cross-Section Properties of Double-Negative and Single- Negative Metamaterials", . April 2004.}

NOTE: At the time of publication, author Andrea Alù was affiliated with the University of Roma Tre. As of September 2006, she is a staff member of the Electrical and Systems Engineering Department at the University of Pennsylvania. Copyright 2004 IEEE. Reprinted from Proceedings of the 2004 IEEE Radar Conference, pages 91-93. Publisher URL: http://ieeexplore.ieee.org/xpl/tocresult.jsp?isNumber=29174\&page=2

This material is posted here with permission of the IEEE. Such permission of the IEEE does not in any way imply IEEE endorsement of any of the University of Pennsylvania's products or services. Internal or personal use of this material is permitted. However, permission to reprint/republish this material for advertising or promotional purposes or for creating new collective works for resale or redistribution must be obtained from the IEEE by writing to pubs-permissions@ieee.org. By choosing to view this document, you agree to all provisions of the copyright laws protecting it.

This paper is posted at ScholarlyCommons. https://repository.upenn.edu/ese_papers/58

For more information, please contact repository@pobox.upenn.edu. 


\title{
Peculiar Radar Cross-Section Properties of Double-Negative and Single-Negative Metamaterials
}

\author{
Abstract \\ Here, we give an overview of some of the results of our analysis of anomalous scattering phenomena for \\ structures involving metamaterial layers, and we provide some physical insights and ideas for potential \\ applications.
}

\section{Keywords}

electromagnetic wave scattering, radar cross-sections, anomalous scattering phenomena, doublenegative metamaterials, inhomogeneous media, metamaterial layers, negative permeability, negative permittivity, radar cross-section properties, single-negative metamaterials

\section{Comments}

NOTE: At the time of publication, author Andrea Alù was affiliated with the University of Roma Tre. As of September 2006, she is a staff member of the Electrical and Systems Engineering Department at the University of Pennsylvania. Copyright 2004 IEEE. Reprinted from Proceedings of the 2004 IEEE Radar Conference, pages 91-93.

Publisher URL: http://ieeexplore.ieee.org/xpl/tocresult.jsp?isNumber=29174\&page=2

This material is posted here with permission of the IEEE. Such permission of the IEEE does not in any way imply IEEE endorsement of any of the University of Pennsylvania's products or services. Internal or personal use of this material is permitted. However, permission to reprint/republish this material for advertising or promotional purposes or for creating new collective works for resale or redistribution must be obtained from the IEEE by writing to pubs-permissions@ieee.org. By choosing to view this document, you agree to all provisions of the copyright laws protecting it. 


\title{
Peculiar Radar Cross-Section Properties of Double- Negative and Single-Negative Metamaterials
}

\author{
Andrea Alù ${ }^{1}$, and Nader Engheta ${ }^{2 *}$ \\ ${ }^{1}$ University of Roma Tre, Department of Applied Electronics \\ via della Vasca Navale, 84 - 00146 Rome, Italy \\ alu@uniroma3.it, http://www.dea.uniroma3.it/lema/people/andrea_alù.html \\ ${ }^{2}$ University of Pennsylvania, Department of Electrical and Systems Engineering \\ Philadelphia, Pennsylvania 19104, U.S.A. \\ engheta@ee.upenn.edu, http://www.ee.upenn.edu/ engheta
}

\begin{abstract}
Here, we give an overview of some of the results of our analysis on anomalous scattering phenomena for structures involving metamaterial layers, and we provide some physical insights and ideas for potential applications.
\end{abstract}

\section{INTRODUCTION}

Metamaterials, i.e., materials with unusual electromagnetic properties that may be constructed by embedding suitably designed inclusions into host media, have attracted a great deal of attention in recent years. In particular, a class of metamaterials, known as the double-negative (DNG) or lefthanded (LH) media, in which both permittivity and permeability have negative real parts at a given frequency, have become the subject of study in various research groups worldwide (see, e.g., [1]-[4]). In one of our previous works [4], we theoretically introduced the idea of ultra-thin subwavelength one-dimensional (1-D) cavity resonators in which a layer of DNG medium was paired with a parallel slab of conventional medium (i.e., "double-positive (DPS)" medium). In that work, we showed that the dispersion relation of such a cavity would not depend on the sum of thicknesses of the interior materials filling the cavity, as it happens for standard cavities filled with conventional (i.e., DPS) materials, but instead it depends on their ratio. We later extended this work to the analyses of parallel-plate waveguides containing a pair of DPS and DNG layers [5][6], guided modes in open DNG slab waveguides, and mode coupling between open DNG and DPS slab waveguides [7]. We have also verified analogous phenomena in studying materials with negative permittivity only ("epsilon-negative", ENG) or negative permeability only ("mu-negative", MNG) [6], [8].

Here in this work, we present a review of some of the unconventional properties of these materials in various scattering scenarios, showing how the electromagnetic wave interaction with suitably designed structures containing metamaterials could lead to interesting results with various potential applications in several areas including radar.

\section{PLANAR CONJUGATE SLABS}

A first case of such scattering anomalies is exhibited in pairing two planar slabs of media with "conjugate" properties, for instance, standard DPS media with DNG metamaterials, or ENG media with MNG materials [8]. Although the wave interaction with each slab by itself has its usual features, it can be shown that the juxtaposition and judiciously pairing of such conjugate slabs may lead to an anomalous response, revealing resonance, complete tunneling, zero reflection and total transparency. A condition on the bilayer parameters for achieving these phenomena for a given plane wave excitation is derived and a more stringent condition for having "total transparency" for a propagating wave with any angle of incidence and for an evanescent wave with the same frequency impinging on the bilayer is also found [8]. It is worth noting that such conditions do not depend on the surrounding environment in which the bilayer is put, but only on the materials employed, since they are based on an "interface resonance" present on the plane interface between the two layers. Using equivalent transmission-line models, further insights and a justification for the field behavior in these resonant pairs of structures are given, and salient features such as the sensitivity to the material parameters, slab thicknesses, dissipation, and angle of incidence are explored.

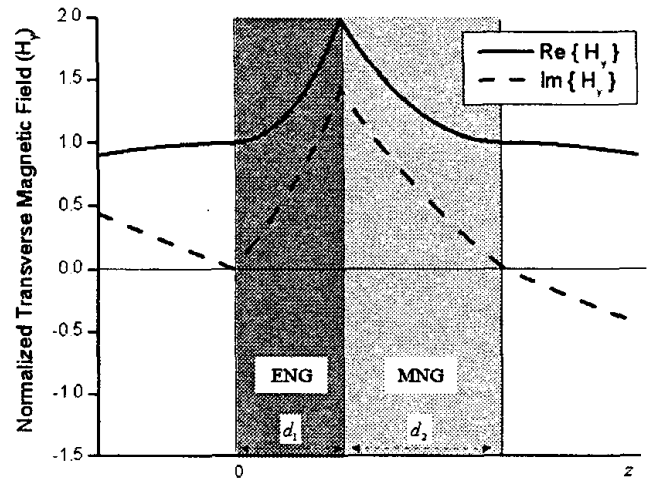

Fig.1 - Total transmission through a planar bi-layer of "conjugate" slabs. The plot shows the real and imaginary parts of total magnetic field when a TM wave illuminates this bilayer.

Figure 1 shows an example of total transmission through an opaque pair of slabs, showing the field distribution typical of this resonant phenomenon.

\footnotetext{
${ }^{*}$ To whom the correspondence should be addressed
} 


\section{TRANSMISSION ENHANCEMENT THROUGH A SUB-WAVELENGTH HOLE IN A PERFECTLY CONDUCTING SCREEN}

Another class of problems somewhat related to the above topic is the role of metamaterials in increasing the wave transmission through sub-wavelength apertures in flat opaque perfectly electric conducting (PEC) screens. We have shown theoretically how the diffraction of wave by a subwavelength aperture on a flat metallic wall may be significantly modified in terms of its radiation pattern and diffracted power, by covering the region around the aperture with suitably designed homogeneous metamaterial layers [9]. This phenomenon can be explained in terms of the leakywave properties of the metamaterial layers. An example of the field distribution in such a case at the resonance is depicted in Figure 2, underlining the concentration of the magnetic field on the hole leading to the enhanced power transmission.

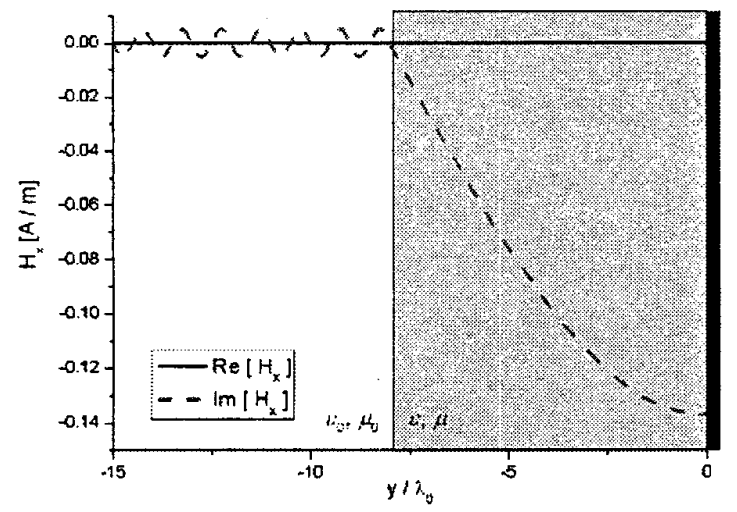

Fig.2 - Magnetic field distribution in front of the hole (in the illuminated side) when the hole is covered with a metamaterial slab designed to enhance the total transmission.

\section{ANOMALOUS SCATTERING CROSS SECTION IN TINY CYLINDRICAL AND SPHERICAL SHELLS}

Some of the peculiar scattering properties of metamaterials can also be investigated in problems involving coaxial shells made of DNG and/or SNG metamaterials. For instance, when we have an electrically thin cylindrical scatterer made of a conventional dielectric, it is well known that the radar cross section (or scattering width) of such a structure is low due to its electrically small radius. However, if the same structure with the same radius is formed by a pair of DNGDPS coaxial layers (or a pair of ENG-MNG coaxial layers), a condition for the existence of anomalous scattering resonance peaks may be obtained. We have theoretically shown that the scattering coefficients may reach its resonant peak when specific ratios of the shell radii for this coaxial structure are achieved [10], and this in principle does not depend on the value of the outer radius. In other words, although the circular cross section of such DPS-DNG or ENG-MNG coaxial shells may be electrically very thin, its scattering width may become significantly large for certain specific ratio of radii. This is consistent with the phenomenon of plasmonic resonance for metallic nanoparticles in the optical and infra-red (IR) regimes. This feature may lead to interesting possibilities for the design of sub-wavelength resonant scatterers whose physical cross sections are much smaller than the wavelength of operation, but for certain applications their scattering widths need to be large. It is also important to note that another set of ratio of radii for these DNG-DPS or ENG-MNG coaxial shells may provide certain opposite effects, namely, low scattering cross section, which can lead to reduction of radar cross section.

Similar phenomena can also be shown in the scattering from sub-wavelength concentric spheres. As in the cylindrical case, a suitably designed pairing of conjugate metamaterials may induce a significant increase in the radar cross section of electrically small concentric spheres made of proper combinations of metamaterials. Our theoretical analysis has shown, for instance, how an electrically small dielectric sphere, which conventionally has a small radar cross section, can provide very large scattering cross section when it is coated by some SNG metamaterials. Conversely, our theoretical investigation has revealed that for a dielectric sphere with certain scattering cross section, one may be able to reduce its cross section by putting an appropriate concentric layer of metamaterial layer either inside or outside the sphere.

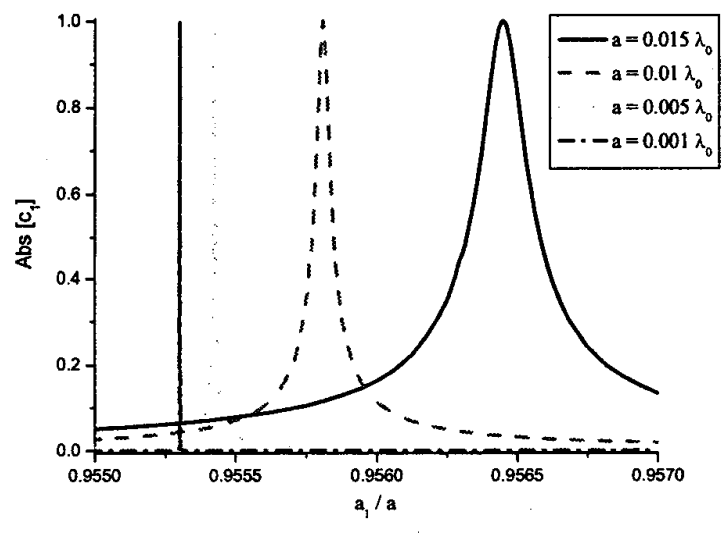

Fig.3 - Scattering peaks in tiny spherical particles with metamaterials.

Figure 3 shows, as an example, the scattering peaks achievable with a suitable pairing of metamaterial shells in a tiny spherical particle.

\section{CONCLUSIONS}

In this work, we present a review of some of our theoretical results on unconventional scattering properties of structures containing metamaterial layers, and provide some physical insights into these findings.

\section{REFERENCES}


[1] V. G. Veselago, "The electrodynamics of substances with simultaneously negative values of $\varepsilon$ and $\mu$," Soviet Physics Uspekhi, 10, 4, pp. 509$514,1968$.

[2] R. W. Ziolkowski, and E. Heyman, "Wave propagation in media having negative permittivity and permeability," Phys. Rev. E., vol. 64, no. 5, $056625,2001$.

[3] R. A. Shelby, D. R. Smith, and S. Schultz, "Experimental verification of a negative index of refraction," Science, vol. 292, no. 5514, pp. 77-79, 2001

[4] N. Engheta, "An idea for thin subwavelength cavity resonators using metamaterials with negative permittivity and permeability," IEEE Antennas and Wireless Propagation Lett., vol. 1, 1, pp. 10-13, 2002.

[5] A. Alù and N. Engheta, "Mono-modal waveguides filled with a pair of parallel epsilon-negative (ENG) and mu-negative (MNG) metamaterial layers," the Digest of IEEE MTT Int. Microwave Symp. (IMS'03), Philadelphia, PA, June 8-13, 2003, pp. 313-316, TU2D-2.

[6] A. Alù and N. Engheta, "Guided modes in a waveguide filled with a pair of single-negative (SNG), double-negative (DNG) and/or doublepositive (DPS) media," IEEE Transactions on Microwave Theory and Techniques, vol. 52, no. 1, pp. 199-210, January 2004.

[7] A. Alù, and N. Engheta, "Anomalous mode coupling in guided-wave structures containing metamaterials with negative permittivity and permeability," Proc. 2002 IEEE-Nanotechnology, Washington DC, August 26-28, 2002, pp. 233-234

[8] A. Alù, and N. Engheta, "Pairing an epsilon-negative slab with a munegative slab: resonance, tunneling and transparency," IEEE Trans. on Antennas and Propagation, Special issue on "Metamaterials", Vol. 51, No. 10, pp. 2558-2571, Oct. 2003.

[9] A. Alu, F. Bilotti, N. Engheta, and L. Vegni, "How Metamaterials May Significantly Affect the Wave Transmission through a Sub-Wavelength Hole in a Flat Perfectly Conducting Screen," Workshop on Metamaterials for Microwave and (Sub) millimetre Wave Applications: Photonic Bandgap and Double Negative Designs, Components and Experiments, London, UK, November 24, 2003.

[10] A. Alu and N. Engheta, "Resonances in Sub-wavelength Cylindrical Structures Made of Pairs of Double-Negative and Double-Negative or Epsilon-Negative and Mu-Negative Coaxial Shells," the special session on "Metamaterials: Double-Negative Structures" in the International Conference in Electromagnetics and Advance Applications (ICEAA' 03) Torino, Italy, September 8-12, 2003, pp. 435-438. 